\title{
Etiological factors of traumatic peripheral nerve injuries
}

Filiz Eser, Lale Akbulut Aktekin, Hatice Bodur, Çiğdem Atan

Department of Physical Medicine and Rehabilitation, Ankara Numune Training and Research Hospital, Samanpazarl, Ankara, Turkey

\begin{abstract}
Background: Traumatic injury of peripheral nerves is a worldwide problem and can result in significant disability. Management of peripheral nerve injuries (PNIs) requires accurate localization and the assessment of severity of the lesion. Aim: The purpose of this study is to analyze the data of patients with PNIs referred for electromyography to a tertiary care hospital. Materials and Methods: This is a retrospective study of clinical and electromyographic data of patients with PNIs seen over a period of eight-years (1999-2007) in a tertiary hospital. The data collected included: Demographic data, cause, type of lesion, anatomical location of the lesion, and the mechanism of lesion. Results: During the study period 938 patients were seen with nerve injuries and the distribution of nerve injuries was: PNIs: 1,165; brachial plexus lesions: 76; and lumbar plexus lesions: 7. The mean age was 31.8 years (range 2-81 years) and the male to female ratio was 2.4:1. The most frequent nerve injuries were ulnar nerve in the upper extremity and sciatic nerve in the lower extremity. The most common cause of nerve injury was motor vehicle accidents. Two-thirds of the PNIs were partial. Conclusion: This study can serve as a guide to determine the epidemiology and classification of traumatic peripheral and plexus injuries.
\end{abstract}

DOI: $10.4103 / 0028-3886.55614$

Key words: Peripheral nerves, electromyography, brachial plexus, lumbosacral plexus

\section{Introduction}

Traumatic injury to peripheral nerves is a worldwide problem and can result in significant disability. ${ }^{[1]}$ The etiological and epidemiological factors of peripheral nerve injuries (PNIs) may vary according to periods of peace or conflict and also according to the development level of populations. Historically, mostly the knowledge of PNIs was developed during wartimes. Traumatic nerve injuries results in significant neurological deficits. In periods of peace, PNIs generally result from motor vehicle accidents (MVAs), lacerations with sharp objects, penetrating trauma, stretching or crushing trauma and fractures, and gunshot wounds. ${ }^{[1]}$ The reported incidence of PNIs among the trauma population was $2 \%$ and $2.8 \%$ and it was $5 \%$ if plexus and root lesions were included. ${ }^{[2,3]}$

Electromyography (EMG) is the most important diagnostic method for evaluating PNIs. These studies help in precise localization of the lesion and also in assessing the severity of the lesion, thus facilitating the treatment options. ${ }^{[1]}$ The purpose of this study was to analyze data of patients with PNIs referred for EMG.

\section{Materials and Methods}

Charts of 9,000 EMG studies performed in patients with PNIs between 1999 and 2007, in the Physical Medicine and Rehabilitation Clinic in a tertiary referral hospital were the subject of the study. The EMG Laboratory of Physical Medicine and Rehabilitation Clinic is a referral clinic for EMG studies for both outpatients and inpatients of the orthopedics, plastic and reconstructive surgery, and physical medicine and rehabilitation clinics of the hospital. Mostly the patients are civilians and come from all over Turkey as the hospital is the major referral center under Ministry of Health and is located in the capital, Central Anatolia. PNI was defined as an injury to a major nerve in either upper or lower extremity and also brachial or lumbar plexus. 
Chart review included identification of PNI and also verification of the database information. The results of the first electrophysiologic studies were considered for the analysis. The data collected included demographic data, the nerve injured, cause of injury, and the severity of injury. All patients had detailed neurologic examination. Motor and sensory conduction studies and needle examination were done by standard techniques using Neuropack 2-MEB 7102-K 2 channels EMG-EP machine (Nihon Kohden Corp. Tokyo, Japan). Electrophysiological involvement was graded as total or partial. Absence of recruitable motor unit potentials on needle examination in the affected muscles was considered as complete nerve injury, (axonotmesis) and partial nerve injury was considered when the EMG studies were suggestive of neurotmesis or total conduction block. Descriptive statistics used included means and frequencies.

\section{Results}

During the study period 938 patients with nerve injuries were seen: PNIs: 1,165; brachial plexus injuries 76; and lumbar plexus injuries seven. Patient population included: 666 men $(71 \%)$ and 272 women $(29 \%)$. The mean age of the patients was $31.8 \pm 17.4$ years (range 2 to 81 years) [Figure 1]. The distribution of nerve injuries, was $77 \%$ in the upper limbs and $23 \%$ in the lower limbs. Of the patients with PNIs, 652 (76\%) patients had single nerve lesion, 118 (14\%) had two nerve lesions, 68 (8\%) three nerve lesions, and $18(2 \%)$ had four or more nerve lesions. The side distribution of nerve injuries was: Right side in $686(54.96 \%)$ patients and left side in 562 (45.04\%). Two-thirds of PNIs were partial and $33.4 \%$ total. The most frequent nerve injuries were: Ulnar nerve $(337,27 \%)$ in the upper limb and sciatic nerve $(138,11.1 \%)$ in the lower limb. Of the 83 plexus injuries, 76 were brachial plexus and seven lumbar plexus lesions. One patient with schizophrenia had bilateral brachial plexus traction injury during one of the violent behavior episode The frequencies of the nerve injuries are given in Table 1. The common causes of nerve injuries were due to MVA and fall related bone fractures and dislocation.
Etiological factors of injuries are summarized in Table 2. Approximately $11 \%$ of lesions were due to an iatrogenic factor, the most common of which was intramuscular (IM) injection [Table 3].

\begin{tabular}{lcc}
\hline \multicolumn{2}{l}{ Table I: Injury distribution according to nerve } & \\
\hline Nerve & $\mathrm{n}$ & $\%$ \\
\hline Ulnar & 337 & 27.00 \\
Median & 273 & 21.9 \\
Radial & 262 & 21 \\
Sciatic & 138 & 11.1 \\
Peroneal & 85 & 6.8 \\
Brachial plexus & 76 & 6.1 \\
Femoral & 34 & 2.7 \\
Axillary & 14 & 1.1 \\
Tibial & 8 & 0.6 \\
Lumbar plexus & 7 & 0.6 \\
Musculocutaneous & 7 & 0.6 \\
Long thoracic & 3 & 0.2 \\
Suprascapular & 2 & 0.2 \\
Accessory & 2 & 0.2 \\
\hline
\end{tabular}

Table 2: Etiological factors of nerve injuries

\begin{tabular}{lccc}
\hline Etiological factor & $\begin{array}{c}\text { Peripheral } \\
\text { nerves (\%) }\end{array}$ & $\begin{array}{l}\text { Brachial } \\
\text { plexus }\end{array}$ & $\begin{array}{c}\text { Lumbar } \\
\text { plexus }\end{array}$ \\
\hline MVA & $252(26.9)$ & $27(2.9)$ & $3(0.3)$ \\
Sharp and & $151(16.1)$ & $2(0.2)$ & - \\
penetrative objects & $142(15.1)$ & - & - \\
Glass wounds & $99(10.6)$ & $4(0.4)$ & $2(0.2)$ \\
latrogenic & $71(7.6)$ & $14(1.5)$ & $2(0.2)$ \\
GSW & $69(7.4)$ & - & - \\
Fall & $41(4.4)$ & $26(2.8)$ & - \\
Traction and blunt & & - & - \\
trauma & $31(3.3)$ & - & - \\
Burn & - & $2(0.2)$ & - \\
Radiotherapy & &
\end{tabular}

MVA - Motor vehicle accidents, GSW - Gunshot wound

Table 3: Distribution of iatrogenic factors

latrogenic factor

n (\%)

Intramuscular injection

Total hip prosthesis operation

$41(41.4)$

Excision of tumor

Tourniquet

Traction or pressure during operation

Intravenous catheterization

Traction during schizophrenic attack

Radiotherapy

Shoulder prothesis
$24(24.3)$

$12(12.1)$

$8(8.1)$

$7(7.1)$

$2(2.0)$

$2(2.0)$

$2(2.0)$

$1(1.0)$

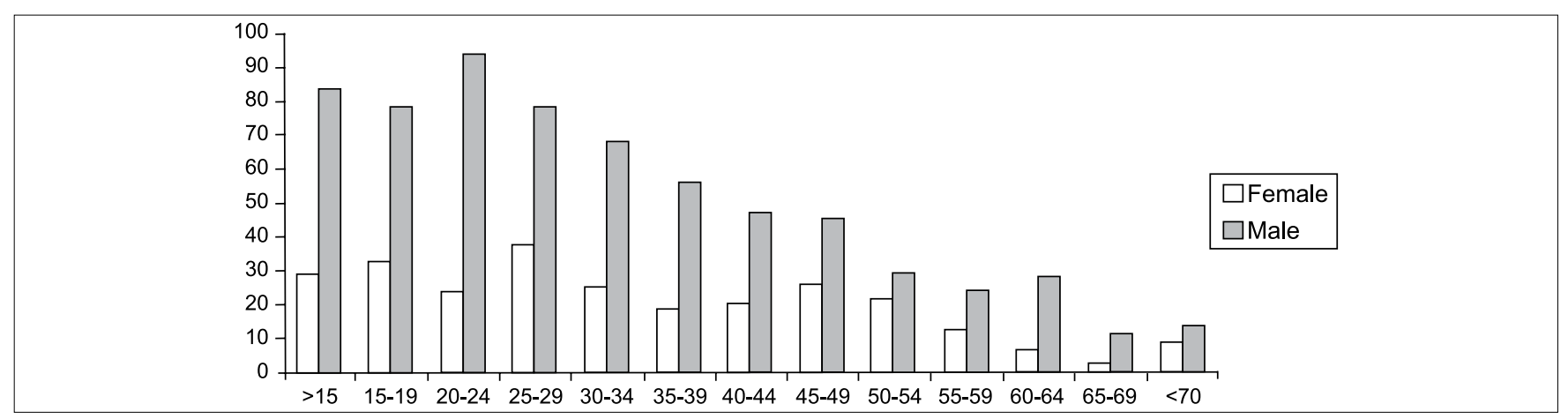

Figure 1: Age and sex distribution of peripheral nerve injury patients 


\section{Discussion}

In PNIs accurate localization of the lesion and assessment of the severity of the lesion were of importance in selecting the appropriate management strategies. ${ }^{[4]}$ By early diagnosis optimal results can be obtained in up to $50 \%$ of injuries with appropriate surgical and conservative treatment. ${ }^{[2]}$ Seddon and Sunderland ${ }^{[5,6]}$ classified nerve injuries into three categories, neuropraxia axonotmesis and neurotmesis. In EMG practice, it is often difficult to place nerve injuries into these classifications. Pure neurapraxia is rare in nerve injury. A majority of partial nerve lesions (the presence of partial clinical and electrophysiological functions of distal nerve lesion) fit best under the description 'partial axonotmesis'. ${ }^{[4]}$ Findings of EMG studies early in the course may be some what similar in complete axonotmesis and neurotmesis. ${ }^{[1]}$ Only follow-up EMG evaluation may differentiate between complete axonotmesis and neurotmesis. ${ }^{[4]}$ By the classification we adopted, partial or total nerve injuries, we could classify the nerve lesions in our patients into partial $66.6 \%$ and complete $33.4 \%$.

Similar to the earlier studies, in this study also the nerve injuries were seen predominantly in young men and in the most productive age group ${ }^{[2,7-9]}$ Trauma-related PNIs were clearly less common after the age of seventy. PNIs may result in considerable morbidity if not managed efficiently, particularly so in the most productive age group..$^{[2,7-10]}$ Nerve injuries are reported more commonly in the upper limbs and the most affected nerves are ulnar, radial and digital nerves. ${ }^{[2,7,9,10]}$ Ulnar nerve was the most frequent nerve lesion in our patients. In the series by Noble et al., ${ }^{[2]}$ radial nerve was the most frequent involved nerve. In their series the main etiological factor was MVAs and lacerations with sharp objects accounted for a small proportion of cases. ${ }^{[2,9]}$ McAllister et al., ${ }^{[10]}$ analyzed upper limb PNIs, and reported digital nerves as the most commonly injured nerves and injuries related to sharp objects as the most frequent cause. However, their patient population was selected from hand surgery units. Other reports showed ulnar nerve injuries as the most frequent nerve injury in the upper limbs. ${ }^{[7,9]}$ In lower limbs peroneal and sciatic nerves were the most frequently involved nerves. ${ }^{[7,9,10]}$

In this series the most common etiological factor was musculoskeletal trauma and the most frequent cause of skeletal trauma was MVAs. Similar were the observations in other series. ${ }^{[7,9,10]}$ The nerve injury in this clinical setting may be related to direct trauma or stretch. The second most frequent cause of PNIs was lacerations with sharp objects. Penetrating trauma with sharp or blunt objects usually results in transection or laceration of the nerve and an immediate nerve reconstruction is indicated in PNIs with sharp nerve lacerations. ${ }^{[2]}$ In our study, iatrogenic factors accounted for $11.2 \%$ of the etiology. About half of the sciatic nerve injuries were related to intragluteal injections or hip arthroplasty. Of the 138 sciatic nerve lesion, 41 were related to intramuscular injection and 24 were related to hip arthroplasty. Earlier studies also reported similar observations in sciatic nerve lesions. ${ }^{[11]}$ In developing countries, nerve lesions caused by unsafe intramuscular injections are still common. The World Health Organization (WHO) reported injections as one of the most common healthcare procedures, and unsafe injections as being associated with morbidity and mortality. ${ }^{[12]}$ The mechanisms of injection-related nerve injury include direct needle trauma, nerve ischemia, circumferential construction by the scar, and direct nerve fiber damage by neurotoxic agents. ${ }^{[13,14]}$ PNI by injections is an iatrogenic tragedy. During the injection procedure, important factors include needle entrance point, needle size, and entrance angle. Although precaution may be taken with routine procedural techniques, complication may still occur, especially in children. ${ }^{[15]}$ Hence, intragluteal injection should avoided in children ${ }^{[16]}$ Gunshot wounds was the cause PNIs in $83.3 \%$ of injuries in a study from Pakistan and in $7.4 \%$ in a study from Canada ${ }^{[2,17]}$ and gunshot wounds accounted for $9.3 \%$ of nerve injuries in our study. Bullets may cause direct damage to the nerve or indirect injury with cavitation effect. ${ }^{[18]}$ The incidence and the etiological spectrum of PNIs reflects the country's development level. In developed countries, MVA is the most common etiological factor. MVA was the most common cause of brachial plexus and lumbar plexus injuries. The other factor included traction and pressure for brachial plexus and gunshot wounds for lumbar plexus lesions. Gunshot wound was the other causative factor for brachial plexus lesions. Gunshot wounds to the plexus are of importance as they may be life-threatening and may lead to extremity loss in addition to causing permanent neurological deficit. ${ }^{[18,19]}$

The major strength of our study is that it is one of the large series of PNIs in our country and reflects the the pattern of nerve injuries in the civilians. The limitations of this study are that it is a retrospective chart analysis and EMG evaluation was done by five different physicians over a period of eight years. EMG studies were done at varying times after the trauma and lesion severity was not specified for all the patients.

PNIs can contribute to major social and economic burden since they generally occur in the most productive age group, the young population. Functional disability associated with nerve injuries can be devastating. Electromyographic evaluation 
and detailed clinical examination of patients with trauma are of great importance in avoiding the nerve injury-related disability.

\section{References}

1. Robinson LR. Traumatic injury to peripheral nerves. Muscle Nerve 2000;23:863-73.

2. Noble J, Munro CA, Prasad VS, Midha R. Analysis of upper and lower extremity peripheral nerve injuries in a population of patients with multiple injuries. J Trauma 1998;45:116-22.

3. Selecki BR, Ring IT, Simpson DA, Vanderfield GK, Sewell MF. Trauma to the central and peripheral nervous systems: Part II: A statistical profile of surgical treatment New South Wales 1977. Aust N Z J Surg 1982;52:111-6.

4. Oh S.J. Traumatic peripheral nerve injuries. In: Clinical electromyography nerve conduction studies. Philadelphia: Williams and Wilkins; 2003. p. $803-19$.

5. Sunderland S. Nerves and nerve injuries, $2^{\text {nd }}$ edition. New York: Churchill Livingston; 1978. p. 133-8.

6. Seddon HJ. Neurovascular injuries. In: Surgical disorders of the peripheral nerves. $2^{\text {nd }}$ edition. Edinburgh, etc: Churchill Livingstone; 1975. p. 89-111.

7. Adeyemi-Doro HO. Pattern of peripheral traumatic neuropathy of the upper limb in Lagos, Injury 1988;19:329-32.

8. Midha R. Epidemiology of brachial plexus injuries in a multitrauma population. Neurosurgery 1997;40:1182-8; discussion 8-9.
9. Kouyoumdjian JA. Peripheral nerve injuries: A retrospective survey of 456 cases. Muscle Nerve 2006;34:785-8.

10. McAllister RM, Gilbert SE, Calder JS, Smith PJ. The epidemiology and management of upper limb peripheral nerve injuries in modern practice. J Hand Surg Br 1996;21:4-13.

11. Plewnia C, Wallace C, Zochodne D. Traumatic sciatic neuropathy: A novel cause, local experience, and a review of the literature. J Trauma 1999;47:986-91.

12. Hutin YJ. Injection safety: A global challenge. Bull World Health Organ $1999 ; 77: 7,8$.

13. Napiontek M, Ruszkowski K. Paralytic drop foot and gluteal fibrosis after intramuscular injections. J Bone Joint Surg Br 1993;75:83-5.

14. Villarejo FJ, Pascual AM. Injection injury of the sciatic nerve (370 cases). Childs Nerv Syst 1993;9:229-32.

15. Bergeson PS, Singer SA, Kaplan AM. Intramuscular injections in children. Pediatries 1982;70:944-8.

16. Mayer M, Romain O. Sciatic paralysis after a buttock intramuscular injection in children: An ongoing risk factor. Arch Pediatr 2001;8:321-3.

17. Babar SM. Peripheral nerve injuries in a third world country. Cent Afr J Med 1993;39:120-5.

18. Samardzic MM, Rasulic LG, Grujicic DM. Gunshot injuries to the brachial plexus. J Trauma 1997;43:645-9.

19. Stewart MP, Birch R. Penetrating missile injuries of the brachial plexus. J Bone Joint Surg Br 2001;83:517-24.

Accepted on 02-07-2009

Source of Support: Nil, Conflict of Interest: None declared. 